\title{
Optimizing mevalonate pathway for squalene production in
}

\section{Yarrowia lipolytica}

\author{
Huan Liu ${ }^{1,2}$, Fang Wang ${ }^{2}$, Li Deng ${ }^{2 *}$ and Peng $\mathrm{Xu}^{1 *}$ \\ ${ }^{1}$ Department of Chemical, Biochemical and Environmental Engineering, University of Maryland \\ Baltimore County, Baltimore, MD 21250. \\ ${ }^{2}$ College of Life Science and Technology, Beijing University of Chemical Technology, Beijing, \\ China.
}

* Corresponding author Tel: +1(410)-455-2474; fax: +1(410)-455-1049. E-mail address: dengli@mail.buct.edu.cn (Li Deng) and pengxu@umbc.edu (PX). 


\begin{abstract}
Squalene is the gateway molecule for triterpene-based natural products and steroidsbased pharmaceuticals. As a super lubricant, it has been used widely in health care industry due to its skin compatibility and thermostability. Squalene is traditionally sourced from sharkhunting or oil plant extraction, which is cost-prohibitive and not sustainable. Reconstitution of squalene biosynthetic pathway in microbial hosts is considered as a promising alternative for cost-efficient and scalable synthesis of squalene. In this work, we reported the engineering of the oleaginous yeast, $Y$. lipolytica, as a potential host for squalene production. We systematically identified the bottleneck of the pathway and discovered that the native HMG-CoA reductase led to the highest squalene improvement. With the recycling of NADPH from the mannitol cycle, the engineered strain produced about $180.3 \mathrm{mg} / \mathrm{l}$ and $188.2 \mathrm{mg} / \mathrm{L}$ squalene from glucose or acetate minimal media, respectively. By optimizing the $\mathrm{C} / \mathrm{N}$ ratio, controlling the media $\mathrm{pH}$ and mitigating the acetyl-CoA flux competition from lipogenesis, the engineered strain produced about $502.7 \mathrm{mg} / \mathrm{L}$ squalene in shake flaks, a 28 -fold increase compared to the parental strain (17.2 $\mathrm{mg} / \mathrm{L})$. We also profiled the metabolic byproducts citric acid and mannitol level and observed that they are reincorporated into cell metabolism at the late stage of fermentation. This work may serve as a baseline to harness $Y$. lipolytica as an oleaginous cell factory for production of squalene or terpene-based chemicals.
\end{abstract}

Key words : Oleaginous yeast, metabolic engineering, mevalonate pathway, squalene, mannitol cycle 


\section{Introduction}

Yarrowia lipolytica is an industrial oleaginous yeast that has been extensively engineered to synthesize lipophilic compounds, including lipids (Qiao, Wasylenko, Zhou, Xu, \& Stephanopoulos, 2017), oleochemicals (P. Xu, Qiao, Ahn, \& Stephanopoulos, 2016), carotenoids (Gao et al., 2017; Macarena Larroude et al., 2018), terpenoids (Jin, Zhang, Song, \& Cao, 2019) and aromatic polyketides (Lv, Marsafari, Koffas, Zhou, \& Xu, 2019) et al. The lipogeneity of this yeast makes it a superior host to produce chemicals that are derived from acetyl-CoA, malonyl-CoA, HMG-CoA and NADPHs. The compartmentalization of oil droplets into lipid bodies provides a hydrophobic environment to sequestrate many lipid-related compounds and mitigate the toxicity issues associated with lipophilic membrane damages. In addition, the ease of genetic manipulation, substrate flexibility and robust growth present us tremendous opportunity to upgrade low-value renewable feedstocks to high-value compounds. It has also been recognized as a 'generally regarded as safe' (GRAS) organism (Groenewald et al., 2014) in the food and nutraceutical industry. A large collection of customized genetic toolboxes, including YaliBricks gene assembly (Wong, Engel, Jin, Holdridge, \& Xu, 2017), CRISPR-Cas9 (Bae, Park, Kim, \& Hahn, 2020; Macarena Larroude, Trabelsi, Nicaud, \& Rossignol, 2020) or CRISPR-Cpf1 (Yang, Edwards, \& Xu, 2020) genome editing, Cre-LoxP-based iterative chromosomal integrations (Lv, Edwards, Zhou, \& Xu, 2019), transposon-based mutagenesis (Wagner, Williams, \& Alper, 2018) and Golden-gate cloning (Celińska et al., 2017; Egermeier, Sauer, \& Marx, 2019; M. Larroude et al., 2019), enabled us to rapidly modify its genome and evaluate many metabolic events to explore the catalytic diversity of this yeast beyond its regular portfolio of fatty acids, fatty alcohols, biofuels et al. Recent metabolic engineering effort in this yeast has allowed us to access more specialized secondary metabolites with pharmaceutical 
values, including sesquiterpenes (Marsafari \& Xu, 2020), triterpenoids (Jin et al., 2019) and flavonoids (Lv, Marsafari, et al., 2019; Palmer, Miller, Nguyen, \& Alper, 2020) et al.

Isoprenoids are a large group of natural products with diverse biological functions. An estimation of more than 70,000 isoprenoids, ranging from monoterpenes, sesquiterpenes, diterpenes and triterpenes have been discovered from nature (Moser \& Pichler, 2019). Isoprenoids play a major role in maintaining membrane homeostasis, protein prenylation for subcellular targeting (Palsuledesai \& Distefano, 2015), signal transduction, the deployment of plant defense pathways, and controlling the transcriptional activity of sterol-responsive-elementbinding-proteins (SREBPs) (Shimano, 2001). The yeast-based mevalonate (MVA) pathway starts with acetyl-CoA condensation reactions, proceeds through the reduction of intermediate HMG-CoA via HMG-CoA reductase, which is the rate-limiting step and the molecular target to design many statins-related anti-cholesterol drugs (Xie \& Tang, 2007). The universal five-carbon precursors isopentenyl diphosphate (IPP) and dimethylallyl diphosphate (DMAPP), derived from mevalonate, are condensed to make the farnesyl pyrophosphate (FPP), which later can be diversified to many sesquiterpenes or triterpenes. Squalene is a 30-carbon triterpene hydrocarbon synthesized from the condensation of two FPPs, which serve as the gateway molecule for all triterpenoids with tens of thousands of structural variations. Squalene possess strong antioxidant and anti-inflammatory activity and is widely used in the cosmetic industry as skin-compatible super-lubricant and hydration protectors (Spanova \& Daum, 2011). Squalene emulsions were used as efficient adjuvants to enhance the immune response of certain vaccines (Spanova \& Daum, 2011). Squalene is primarily sourced from shark liver, which poses significant ecological or ethical concerns related with shark-hunting. Reconstitution of squalene pathway in microbes may provide an alternative route to sustainably produce squalene from renewable feedstocks. A 
number of metabolic engineering studies have set the effort to engineer bacteria or bakers' yeast to produce squalene, with improved yield and process efficiency. For example, a recent work identified that yeast peroxisome may serves as a dynamic depot to store squalene up to $350 \mathrm{mg} / \mathrm{g}$ dry cell weight (G.-S. Liu et al., 2020), despite the highly oxidative nature of peroxisome. In this work, we report the systematic optimization and engineering of the endogenous mevalonate pathway in Yarrowia lipolytica for efficient synthesis of squalene from simple synthetic media. We identified the bottlenecks of the mevalonate pathway and discovered alternative reducing equivalents (NADPH) pathways to improve squalene production. Our engineered strain produced up to $502.7 \mathrm{mg} / \mathrm{L}$ of squalene in shake flask. This work may set a foundation for us to explore oleaginous yeast as a chassis for cost-efficient production of squalene and triterpenoids in a long-term run.

\section{Methods and Materials}

\subsection{Strains and culture conditions}

Escherichia coli NEB5 $\alpha$ high efficiency competent cells were obtained from NEB for plasmid construction, preparation, propagation and storage. The Y. lipolytica wild type strain W29 was purchased from ATCC (ATCC20460). The auxotrophic Po1g (Leu-) was obtained from Yeastern Biotech Company (Taipei, Taiwan). All strains and plasmids are listed in supplementary Table S2.

LB broth or agar plate with $100 \mu \mathrm{g} / \mathrm{mL}$ ampicillin was used to cultivate E. coli strains. Yeast rich medium (YPD) was prepared with $20 \mathrm{~g} / \mathrm{L}$ Bacto peptone (Difco), $10 \mathrm{~g} / \mathrm{L}$ yeast extract (Difco), and $20 \mathrm{~g} / \mathrm{L}$ glucose (Sigma-Aldrich), and supplemented with $15 \mathrm{~g} / \mathrm{L}$ Bacto agar (Difco) for solid plates. YNB seed medium was made with $1.7 \mathrm{~g} / \mathrm{L}$ yeast nitrogen base (without amino acids and ammonium sulfate) (Difco), $5 \mathrm{~g} / \mathrm{L}$ ammonium sulfate (Sigma-Aldrich), $0.69 \mathrm{~g} / \mathrm{L}$ CSM- 
Leu (Sunrise Science Products, Inc.) and $20 \mathrm{~g} / \mathrm{L}$ glucose. Selective YNB plates were made with YNB media supplemented with $15 \mathrm{~g} / \mathrm{L}$ Bacto agar (Difco). In fermentation process, YNB fermentation medium with glucose as substrate and carbon/nitrogen molar ratio of 80:1 was made with $1.7 \mathrm{~g} / \mathrm{L}$ yeast nitrogen base (without amino acids and ammonium sulfate) (Difco), 1.1 g/L ammonium sulfate (Sigma-Aldrich), 0.69 g/L CSM-Leu (Sunrise Science Products, Inc.) and $40 \mathrm{~g} / \mathrm{L}$ glucose. YNB fermentation medium with sodium acetate as substrate and carbon/nitrogen molar ratio 80:1 was made with $1.7 \mathrm{~g} / \mathrm{L}$ yeast nitrogen base (without amino acids and ammonium sulfate) (Difco), $0.825 \mathrm{~g} / \mathrm{L}$ ammonium sulfate (Sigma-Aldrich), $0.69 \mathrm{~g} / \mathrm{L}$ CSM-Leu (Sunrise Science Products, Inc.), 41 g/L sodium acetate. Glacial acetic acids were purchased from SigmaAldrich.

Phosphoric buffer solution (PBS) with $\mathrm{pH} 6.0$ was made with $0.2 \mathrm{M} \mathrm{Na} 2 \mathrm{HPO} 4$ and $0.2 \mathrm{M}$ $\mathrm{Na}_{2} \mathrm{HPO}_{4}$, which was used to replace water to make YNB-glucose-PBS fermentation medium. Bromocresol purple was a $\mathrm{pH}$-sensitive indicator which could change its color with the $\mathrm{pH}$ from 5.2-7.0 (El-Ashgar et al., 2012) and $40 \mathrm{mg} / \mathrm{L}$ bromocresol purple was added into fermentation medium to indicate $\mathrm{pH}$ variation. The $\mathrm{pH}$ of medium was regulated to 6.0 by $6.0 \mathrm{M} \mathrm{HCl}$ in the fermentation process. The components in glucose-YNB media with $\mathrm{C} / \mathrm{N}$ ratio 60:1, 40:1, 20:1, 10:1 were as same as them in $\mathrm{C} / \mathrm{N}$ ratio 80:1 except the content of ammonium sulfate changed to $1.47 \mathrm{~g} / \mathrm{L}, 2.2 \mathrm{~g} / \mathrm{L}, 4.4 \mathrm{~g} / \mathrm{L}, 8.8 \mathrm{~g} / \mathrm{L}$ respectively, to explore the effect of $\mathrm{C} / \mathrm{N}$ ratio on squalenen accumulation. And $1 \mathrm{mg} / \mathrm{L}$ cerulenin solution prepared with dimethylsulfoxide (DMSO) was added into fermentation medium to inhibit precursor (fatty acids biosynthesis) competing pathway.

\subsection{Genetic transformation of Y. lipolytica}


All plasmids constructed were transformed into the $Y$. lipolytica host strain Polg $\Delta$ Leu using the lithium acetate/single-strand carrier DNA/PEG method (Chen, Beckerich, \& Gaillardin, 1997). And single fresh Y. lipolytica colonies were picked from YNB selective plates and inoculated into YNB seed media, which were grown at $30^{\circ} \mathrm{C}$ for $48 \mathrm{~h}$. For tube test, $100 \mu \mathrm{L}$ seed cultures were inoculated into $5 \mathrm{~mL}$ fermentation media in $50 \mathrm{~mL}$ tube. $600 \mu \mathrm{L}$ seed cultures were inoculated into $30 \mathrm{~mL}$ fermentation media in $250 \mathrm{~mL}$ shake flasks with $250 \mathrm{rpm}$ and $30{ }^{\circ} \mathrm{C}$. Time series samples were taken for analyzing biomass, sugar content, and squalene titer.

\subsection{Analytical methods}

Four OD units of liquid yeast cell was harvested and subsequently was pelleted by centrifugation at $14,000 \mathrm{rpm}$ for $5 \mathrm{~min}$. Water was completely withdrawn and $500 \mathrm{uL} 0.5 \mathrm{M}$ sodium methoxide (sodium hydroxide dissolved in pure methanol) was used to resuspend the cell pellet. The mixture was kept at room temperature with shaking for 2 hours at 1,200 rpm with a high-duty vortex to allow complete saponification of lipids and extraction of squalene. Then 400 $\mathrm{uL}$ hexane was added to extract squalene. The mixture was vortexed at room temperature for 10 min and hexane phase was directly injected to gas chromatography-FID (GC-FID) for squalene analysis. Gas chromatography-flame ionization detector (GC-FID) system (Agilent 7820A) equipped with HP-5 column $(30 \mathrm{~m} \times 320 \mu \mathrm{m} \times 0.25 \mu \mathrm{m})$ was used to detect squalene, using helium as the carrier gas with a linear velocity of $2 \mathrm{ml} / \mathrm{min}$. The column temperature profile was $175^{\circ} \mathrm{C}$ for $3 \mathrm{~min}, 20{ }^{\circ} \mathrm{C} / \mathrm{min}$ ramping to $200{ }^{\circ} \mathrm{C}$ and holding for $3 \mathrm{~min}$, and then $20{ }^{\circ} \mathrm{C} / \mathrm{min}$ ramping to $260{ }^{\circ} \mathrm{C}$ and holding for $4 \mathrm{~min}$.

The cell growth was monitored by measuring the optical density at $600 \mathrm{~nm}$ (OD600) with a UV-vis spectrophotometer that could also be converted to dry cell weight (DCW) according to the calibration curve DCW : OD600 $=0.33: 1(\mathrm{~g} / \mathrm{L})$. The fermentation broth was centrifuged at 
$14,000 \mathrm{rpm}$ for $5 \mathrm{~min}$ and the supernatant was used for analyzing the concentration of glucose, mannitol, and acetic acid by HPLC with a refractive index detector and Supelcogel TM Carbohydrate column. The column was eluted with $10 \mathrm{mM} \mathrm{H}_{2} \mathrm{SO}_{4}$ at a column temperature of $50{ }^{\circ} \mathrm{C}$, a refractive index detector temperature of $50{ }^{\circ} \mathrm{C}$, and a flow rate of $0.4 \mathrm{~mL} / \mathrm{min}$.

\subsection{Plasmid and pathway construction}

All primers are listed in supplementary Table S1. All restriction enzymes were purchased from Fisher FastDigest enzymes. Plasmid miniprep, PCR clean-up, and gel DNA recoveries were using Zyppy and Zymoclean kits purchased from Zymo research. All the genes were PCRamplified with the primers from genomic DNA of Y. lipolytica, S. cerevisiae, E. coli, B. subtilis, Aspergillus nidulans, respectively (Supplymentary Table S1 and Table S2). All these genes were inserted into downstream of the Y. lipolytica TEF-intron promoter in the pYLXP' vector backbone (Wong et al., 2017) at the SnaBI and KpnI site via Gibson assembly (Gibson et al., 2009). Upon sequence verification by Genewiz, the restriction enzyme AvrII, NheI, NotI, ClaI and SalI (Fermentas, Thermo Fisher Scientific) were used to digest these vectors, and the donor DNA fragments were gel purified and assembled into the recipient vector containing previous pathway components in compliance with the YaliBricks subcloning protocol (Wong et al., 2017; Wong, Holdridge, Engel, \& Xu, 2019). All assembled plasmids were verified by gel digestion and were subsequently transformed into the $Y$. lipolytica host strain Polg $\Delta$ Leu using the lithium acetate/single-strand carrier DNA/PEG method (Chen et al., 1997). In chromosomal integration process, pYLXP' vector assembled with functional genes was linearized by restriction enzyme NotI (Fermentas, Thermo Fisher Scientific). The linear fragment was transformed into the $Y$. lipolytica host strain Po1g $\Delta$ Leu and cultivated on CSM-Leu minimal media (agar plate) for colony screening. The screened colony was later cultivated in YPD media and genomic DNA 
was extracted with Wizard genomic kits (Promega). Then the genomic samples were used as template for PCR verification of the integrated gene with gene-specific primers.

\section{Results and discussions}

\subsection{Debottlenecking mevalonate pathway for squalene production}

In yeast, squalene was primarily synthesized from the mevalonate (MVA) pathway (Fig. 1). Staring with acetyl-CoA condensation, yeast uses a number of critical enzymes to synthesize squalene, including acetoacetyl-CoA thiolase (Erg 10, YALI0B08536g), HMG-CoA reductase (YALI0E04807g), mevalonate kinase (Erg 12, YALI0B16038g), phosphomevalonate kinase (Erg 8, YALI0E06193g), mevalonate pyrophosphate decarboxylase (MVD1, YALI0F05632g), farnesyl pyrophosphate synthase (Erg20, YALI0E05753g), geranyl pyrophosphate synthase (YALI0D17050g) and squalene synthase (SQS1, YALI0A10076g). Genome annotation indicates

that $Y$. lipolytica contains the complete mevalonate pathway (Fig. 1). In MVA pathway, HMGCoA reductase was reported as the rate-limiting metabolic step in squalene accumulation (RODWELL, NORDSTROM, \& MITSCHELEN, 1976). In addition, there was almost no squalene accumulated by native $Y$. lipolytica due to the quick consumption of squalene by downstream ergosterol synthase. After we overexpressed the endogenous squalene synthase gene $(S Q S)$, squalene production was increased to $17.25 \mathrm{mg} / \mathrm{L}$ at $120 \mathrm{~h}$ with chemically-defined complete synthetic media (CSM-leu) in test tube. With this as a starting strain, we investigated the effect of three HMG-CoA reductases (encoded by HMG) on squalene production. The three HMGs were derived from Saccharomyces cerevisiae, Silicibacter pomeroyi and Y. lipolytica. Truncated form of HMG-CoA reductase devoid of N-terminal membrane targeting signal has been proven to be effective in improving isoprenoid production in Saccharomyces cerevisiae (encoded by SctHMG ) (Polakowski, Stahl, \& Lang, 1998; Thompson, Kwak, \& Jin, 2014). 
When co-expressed with endogenous SQS, the strain with the truncated HMG1 (SctHMG) led to squalene production at $83.76 \mathrm{mg} / \mathrm{L}$ (Fig. 2A), indicating that overexpression of HMG-CoA reductase was beneficial for squalene production. To test whether other sources of HMG-CoA reductase could display better functions, we co-expressed SpHMG from Silicibacter pomeroyi and endogenous $y l H M G$ with SQS, respectively. A low yield of squalene $(9.24 \mathrm{mg} / \mathrm{L})$ was produced in the strain expressing $S p H M G$. This result was consistent with previous findings that HMG from Silicibacter pomeroyi was highly specific for NADH (Meadows et al., 2016) and this bacterial-derived enzymes could not be directly translated to yeast system. When endogenous $y l H M G$ was co-expressed with SQS (strain HLYaliSO1), the engineered strain yielded 121.31 $\mathrm{mg} / \mathrm{L}$ squalene at $120 \mathrm{~h}$ in test tube, demonstrating the potential of using $Y$. lipolytica as a platform to synthesize various terpenes. We also tested the truncated form of $y l H M G$ sequence (YALI0E04807p), of which the first 495 nucleotides that encode the 165 amino acid N-terminal domain responsible for membrane localization (ER targeting) were removed. The remaining Cterminal residues containing the catalytic domain and an NADPH-binding region (Gao et al., 2017) were overexpressed. We then overexpressed the truncated $y l H M G$ (t495ylHMG) to compare how the variation of $y l H M G$ may improve squalene synthesis. Contrary to our hypothesis, removal of the N-terminal 495bp of $y l H M G$ exhibits adverse effect on both squalene production and cell growth (Fig. 2A), indicating that the $\mathrm{N}$-terminal membrane-binding domain plays a critical role in squalene synthesis.

In addition to the overexpression of the endogenous SQS and $y l H M G$ genes, we also tested whether the expression of other genes in the MVA pathway would improve squalene production, including ylErg8 encoding phosphomevalonate kinase, ylErg10 encoding acetoacetyl-CoA thiolase, Erg12 encoding mevalonate kinase, and ylErg20 encoding farnesyl 
pyrophosphate synthetase; ylGPS encoding geranyl pyrophosphate synthase. And ylErg8, ylErg10, Erg12 and ylErg20 from S. cerevisiae were also overexpressed to compare how the variation of these genes may enhance squalene synthesis. As shown in Fig. 2B, cooverexpression of ylErg8, ylErg10, Erg12 could not further improve squalene synthesis, regardless of the source of the gene. Among all of these combinations (Fig. 2B), the highest squalene production was obtained for the strain in which ylGPS and SQS-ylHMG1 were overexpressed, with titer of $107.08 \mathrm{mg} / \mathrm{L}$ and a specific production of $36.24 \mathrm{mg} / \mathrm{g}$ DCW, which is still lower than the strain only expressing SQS-ylHMG1. These results indicate that sequential overexpression of the genes involved in the MVA pathway could not further improve the carbon flux toward squalene, possibly due to the stringent regulation of MVA pathway at multiple nodes, including ergosterol-mediated feedback inhibition or SREBP-related transcriptional repression.

\subsection{Augmenting NAPDH and acetyl-CoA precursor pathways to improve squalene production}

$\mathrm{NADPH}$ as the primary biological reducing equivalent protects cell from oxidative stress and extend carbon-carbon backbones, which was also reported as the major rate-limiting precursor in fatty acids synthesis in oleaginous species (Qiao et al., 2017; Wasylenko, Ahn, \& Stephanopoulos, 2015). HMG-CoA reductase (HMGR) is the first rate-limiting enzyme in the mevalonate pathway and plays critical role in regulating squalene biosynthesis (Ma et al., 2019). 3-hydroxy-3-methylglutaryl coenzyme A (HMG-CoA) is reductively hydrolyzed to mevalonate by releasing coenzyme A with NADPH as reducing equivalent (Cao, Wei, Lin, \& Hua, 2017). Based on previous work, source of cytosolic NADPH in the Baker's yeast may originate from various alternative routes depending on the carbon source and genetic background of the yeast 
strain (Huan Liu, Marsafari, Deng, \& Xu, 2019; Minard, Jennings, Loftus, Xuan, \& McAlisterHenn, 1998; Minard \& McAlister-Henn, 2005). With glucose as carbon sources, cytosolic NADPH primarily relies on the pentose phosphate pathway. Other cytosolic NADPH pathways include NADP-specific isocitrate dehydrogenase (IDP2), malic enzyme (ylMAE), mannitol dehydrogenase (ylMnDH1, ylMnDH2), 6-phosphogluconate dehydrogenase (ylGND2) and succinate semialdehyde dehydrogenase (ylUGA2) (Huan Liu, Monireh Marsafari, Li Deng, et al., 2019) (Fig. 1). In this work, we tested a collection of auxiliary cytosolic NADPH pathways and investigated how these pathways may enhance squalene production and cellular fitness on the basis of co-expression SQS-ylHMG (Fig. 3A). Among these chosen NADPHs, mannitol dehydrogenase (ylMnDH2, encoded by YALI0D18964g) presented the best results to improve squalene production. Mannitol, a more reduced sugar alcohol compared to glucose, played an essential role in modulating cytosolic NADPHs through the mannitol cycle. This could partially explain why mannitol was the major byproduct during lipid accumulation phase in $Y$. lipolytica (P. Xu, Qiao, \& Stephanopoulos, 2017). When ylMnDH2 was overexpressed with SQS and ylHMG (strain HLYaliS02, Supplymentary Table S2), the engineered strain produced 11\% more squalene with volumetric production titer increased to $135.22 \mathrm{mg} / \mathrm{L}$, despite relatively decreased yield of $32.33 \mathrm{mg} / \mathrm{g}$ DCW (Fig. 3A). This is possibly ascribed to the increased cell fitness and lipid content after enhancing the supplement of NADPH.

Apart from NADPH, acetyl-CoA, is an essential metabolic intermediate connecting glycolysis, Krebs cycle, and glyoxylate shunt pathways. Acetyl-CoA is also the intermediate metabolite participated in lipid synthesis, peroxisomal lipid oxidation and amino acid degradation pathways. It links both anabolism and catabolism, is the starting molecule in MVA pathway. Cytosolic acetyl-CoA was found as a critical precursor to boost secondary metabolite 
production (Huan Liu, Marsafari, Wang, Deng, \& Xu, 2019). For example, engineering alternative cytosolic acetyl-CoA pathways were proven to be efficient strategies to improve fatty acids and isoprenoid production in both Bakers' yeast and Y. lipolytica (Hu Liu, Fan, Wang, Li, \& Zhou, 2019; Meadows et al., 2016; van Rossum, Kozak, Pronk, \& van Maris, 2016). Therefore, we next investigated whether endogenous and various heterologous acetyl-CoA pathways could improve squalene production. First, we investigated the pyruvate decarboxylase (PDC), acetylaldehyde dehydrogenase (ALD) and acetyl-CoA synthase (ACS) bypass (Fig. 1) and compared the efficiency of this route from Y. lipolytica, S. cerevisiae and E.coli (Fig. 3B). By overexpression of pyruvate decarboxylase (ScPDC) from $S$. cerevisiae and acetylaldehyde dehydrogenase (EcPuuc) from E.coli, we obtained only $106.54 \mathrm{mg} / \mathrm{L}$ of squalene (Fig. 3B). We observed that the cell growth fitness was negatively impacted due to the expression of heterologous genes, possibly due to the accumulation of the toxic aldehyde intermediate. We next attempted the endogenous ATP citrate lyase, which is the primary acetyl-CoA route to $Y$. lipolytica metabolism. ATP citrate lyase (ACL) was mainly used for supply of the cytosolic acetyl-CoA, which was proven to have two isoforms encoded by two separate genes in $Y$. lipolytica (ACL1 and ACL2) (Nowrousian, Kück, Loser, \& Weltring, 2000). Endogenous ylACL1 (YALIOE34793g) and ylACL2 (YALIOD24431g) genes were subsequently tested. A $19.5 \%$ increase in squalene synthesis was obtained in the resulting strains HLYaliSO3 with ylACL2 overexpressed along with SQS and ylHMG, leading to the titer of squalene $144.96 \mathrm{mg} / \mathrm{L}$ (Fig. 3B). The increase was probably a result of the pushing strategies for acetyl-CoA enrichment by expressing ACL2 so that adequate cytosolic acetyl-CoA could be pushed into the MVA pathway for the synthesis of squalene. Surprisingly, the specific yield reduced to 25.27 $\mathrm{mg} / \mathrm{g}$ DCW which was caused by the enhancement of cell growth due to the increased lipid 
content. This increased lipid content may also serve as the storage space to sequestrate squalene in our engineered cell.

\subsection{Glucose and acetate as media for squalene production}

$Y$. lipolytica can grow on a broad range of substrates and convert various organic waste to high-value chemicals (Dobrowolski, Mituła, Rymowicz, \& Mirończuk, 2016; Rakicka, Biegalska, Rymowicz, Dobrowolski, \& Mirończuk, 2017). For example, it has been reported that $Y$. lipolytica possessed strong acetate utilization pathway that is equivalent or even superior to the hexose utilization pathway, which led to an improvement of triacylglyceride (TAG) production from $100 \mathrm{~g} / \mathrm{L}$ to $115 \mathrm{~g} / \mathrm{L}$ in bench-top bioreactors, when the cultivation was switched from glucose media to acetate media (Qiao et al., 2017; J. Xu, Liu, Qiao, Vogg, \& Stephanopoulos, 2017). In another work, $Y$. lipolytica was reported to efficiently uptake acetic acid as sole carbon source to produce polyketides up to $4.76 \mathrm{~g} / \mathrm{L}$, indicating that acetate may serve as a metabolic "shortcut" to acetyl-CoA with improved carbon conversion efficiency and pathway yield (Huan Liu, Monireh Marsafari, Fang Wang, et al., 2019). In this study, a similar strategy was explored to investigate the conversion process of acetate to squalene by the engineered strain HLYaliSO1, HLYaliSO2 and HLYaliS03 (Supplementary Fig. S1). 41 g/L sodium acetate (NaAc), equivalently to $29.5 \mathrm{~g} / \mathrm{L}$ acetic acid (HAc, $0.5 \mathrm{M}$ ) was used to cultivate the engineered strains. In situ $\mathrm{pH}$ indicator (bromocresol purple) was used to track the $\mathrm{pH}$ change and $6 \mathrm{M} \mathrm{HCl}$ was used to adjust the $\mathrm{pH}$ in the shake flask. Among the engineered strains, the highest squalene titer reached $191.68 \mathrm{mg} / \mathrm{L}$ at $168 \mathrm{~h}$ in acetate-YNB medium by strain HLYaliS01, with $99 \%$ of acetic acid depleted and $6.6 \mathrm{~g} / \mathrm{L}$ biomass produced, yielding of squalene at $29.04 \mathrm{mg} / \mathrm{g}$ DCW 
(Supplementary Fig. S1 B and Table S3 ). Strain HLYaliSO2 produced $180.28 \mathrm{mg} / \mathrm{L}$ squalene at $140 \mathrm{~h}$ in acetate-YNB medium with the highest productivity (Supplementary Fig. S1 D). When both engineered strains (HLYaliSO1 and HLYaliSO2) were cultivated in glucose-YNB medium, $157.81 \mathrm{mg} / \mathrm{L}$ and $188.18 \mathrm{mg} / \mathrm{L}$ squalene was achieved by strain HLYaliSO1 and HLYaliSO2, with a yield of $16.53 \mathrm{mg} / \mathrm{g}$ DCW and $15.91 \mathrm{mg} / \mathrm{g}$ DCW, respectively (Supplementary Fig. S1 A and C, Table S3). This indicates that the mannitol cycle (which is engineered in strain HLYaliSO2 with $S Q S-y l H M G-y l M n D H 2)$ may function well when glucose is used as carbon source. Compared with HLYaliSO1 and HLYaliSO2, HLYaliSO3 (the strain with SQS-ylHMG-ylACL2) produced less squalene in glucose $(138.33 \mathrm{mg} / \mathrm{L})$, but similar amount of squalene in acetate $(176.8 \mathrm{mg} / \mathrm{L})$ (Supplementary Fig. S1 E and F). The data demonstrated that both glucose and acetate could be utilized as carbon sources to produced squalene by $Y$. lipolytica and acetate as a potential and cheap industrial chemicals had a promising application and commercial value for squalene and terpene production.

To further improve squalene synthesis, we assembled ylACL2 to the plasmid harboring SQS, ylHMG and ylMnDH2. But the engineered strain (HLYaliSO4) didn't result in an improved squalene production from neither glucose nor acetate as substrate (Supplementary Fig. S2), possibly due to the metabolic imbalance or gene expression overloading causing burdensome effects to the cell factory.

\subsection{Shake flask cultivation of engineered strain with $\mathrm{pH}$ and carbon/nitrogen ratio optimization}

Despite that glucose is the preferred carbon source for cell growth, a similar level of squalene production was detected in our engineered strains (HLYaliSO1 and HLYaliSO2, 
HLYaliS03). Y. lipolytica is a natural lipid producer, accumulating up to $30 \% \sim 60 \%$ cell weight as lipid, which leads to a strong competition for the precursor acetyl-CoA (P. Xu et al., 2017). Meanwhile, cultivation $\mathrm{pH}$ and media $\mathrm{C} / \mathrm{N}$ ratio were two critical factors that affect cellular morphology and growth in Y. lipolytica (Szabo, 1999).

In our previous work, we observed a quick declining of cultivation $\mathrm{pH}$ from 6 to 3.5 in polyketide synthesis, due to the accumulation of citric acid when glucose was utilized. The $\mathrm{pH}$ variations negatively affect strain physiology, alter cell membrane permeability and limit nutrients transportation due to the loss of proton driving force. A significant improvement of polyketide titer was observed by combining PBS buffer with $1 \mathrm{mg} / \mathrm{L}$ cerulenin supplementations. Cerulenin is known to irreversibly form a covalent adduct with the active site (cysteine residue) of $\beta$-ketoacyl-ACP synthase, inhibiting the elongation of the fatty acid backbone (Huan Liu, Monireh Marsafari, Fang Wang, et al., 2019). Thus, a similar strategy was applied to promote squalene production by strain HLYaliSO2 (shown in Fig. 4 A and Supplementary Fig. S3). When the engineered strain was cultivated in the minimal YNB media with $0.2 \mathrm{M}$ phosphoric buffer solution (PBS, pH 6.0), squalene production was increased to $354.44 \mathrm{mg} / \mathrm{L}$ at $168 \mathrm{~h}$ (Supplementary Fig. S3), which was an increase of $88.4 \%$, compared with the results from $\mathrm{pH}$ uncontrolled (Supplementary Fig. S1 C) experiment. The improvement is ascribed to the better growth fitness under $\mathrm{pH}$ control and the biomass of strain HLYaliSO2 reached $13.98 \mathrm{~g} / \mathrm{L} \mathrm{DCW}$ with the squalene specific yield at $25.35 \mathrm{mg} / \mathrm{g}$ DCW (Supplementary Table S3). The major byproduct mannitol accumulated up to $2.2 \mathrm{~g} / \mathrm{L}$ at 48 hour and citric acid reached $7.81 \mathrm{~g} / \mathrm{L}$ at $96 \mathrm{~h}$; both mannitol and citrate were subsequently reincorporated into cell metabolism (Supplementary Fig. S3). But only $\sim 50 \%$ of glucose was utilized in this process which was consistent with our previous work, indicating the supplementation of $\mathrm{PO}_{4}{ }^{3-}$ buffer may negatively impact the glucose 
uptake rate. To further enhance squalene synthesis, $1 \mathrm{mg} / \mathrm{L}$ cerulenin was supplemented to the minimal YNB-PBS media at $48 \mathrm{~h}$ and the squalene production increased to $384.13 \mathrm{mg} / \mathrm{L}$ at $188 \mathrm{~h}$, an $8.4 \%$ increase compared with the result without cerulenin (Fig. 4 A). A similar fermentation profile of glucose consumption, mannitol, and citric acid accumulation was found: half of glucose was utilized while $14.9 \mathrm{~g} / \mathrm{L}$ DCW was obtained with the squalene specific yield at 25.78 mg/g DCW (Supplementary Table S3). Byproduct mannitol reached $1.9 \mathrm{~g} / \mathrm{L}$ at $48 \mathrm{~h}$, but citric acid increased to $9.7 \mathrm{~g} / \mathrm{L}$, which was higher than that in the YNB-PBS media without cerulenin supplemented, possibly due to the fact that inhibition of the endogenous fatty acid synthase may prevent citrate from being converted to acetyl-CoA and oxaloacetate by ATP-citrate lyase (encoded by ACL).

We next investigated the effect of $\mathrm{C} / \mathrm{N}$ ratio on squalene production in YNB-PBS media supplemented with cerulenin (Fig. 4 B). Various C/N ratios including 10:1, 20:1, 40:1, 60:1 and 80:1 was studied (Supplementary Fig. S4). When the $\mathrm{C} / \mathrm{N}$ ratio was set at $60: 1$, a similar fermentation profile of glucose consumption, mannitol, dry cell weight, citric acid was obtained, compared to the metabolic profile for C/N 80:1. Squalene titer at C/N 60:1 reached $396 \mathrm{mg} / \mathrm{L}$ at $120 \mathrm{~h}$ with increased productivity. The highest squalene titer was achieved in the media with $\mathrm{C} / \mathrm{N}$ ratio $40: 1$, reaching $502.75 \mathrm{mg} / \mathrm{L}$ at $120 \mathrm{~h}$ with the yield to $32.6 \mathrm{mg} / \mathrm{g}$ DCW (Fig. $4 \mathrm{~B}$, supplementary Table S3), which was $30.8 \%$ higher than the squalene production form $\mathrm{C} / \mathrm{N}$ ratio 80:1 media. We speculated that acetyl-CoA flux was enlarged and flowed to MVA pathway, since we observed less citric acid accumulation $(1.9 \mathrm{~g} / \mathrm{L})$ at the end of fermentation. However, when the $\mathrm{C} / \mathrm{N}$ ratio was further reduced to $20: 1$ or 10:1, adverse effect was obtained with decreasing squalene production (Supplementary Fig. S4). We speculated that the superfluous nitrogen provision may lead more carbons flowing to cell growth. These results illustrated that 
$\mathrm{C} / \mathrm{N}$ ratio plays an important role in the redistribution of carbon flux and strongly influenced the accumulation of squalene. Further downregulation of acetyl-CoA carboxylase (ACC) may be required to improve squalene production. ACCase, as the malonyl-CoA source pathway and the acetyl-CoA sink pathway during lipogenesis, was primarily controlled through the phosphorylation of serine residues by Snf1-mediated AMP kinase. Inhibition of fatty acid synthase pathway and nitrogen starvation was proven to be effective strategies to activate Snf1 kinase and slows down ACC1 activity (Seip, Jackson, He, Zhu, \& Hong, 2013; Zhang, Galdieri, \& Vancura, 2013). It was consistent with our findings that medium $\mathrm{C} / \mathrm{N}$ ratio was beneficial for squalene synthesis. By applying these engineering strategies, we have obtained an oleaginous yeast strain with a similar squalene level to the strain $S$. cerevisiae (Han, Seo, Song, Lee, \& Choi, 2018; Huang et al., 2018). This work highlights the potential of engineering $Y$. lipolytica as a promising microbial platform for efficient synthesis of squalene and terpene-related compounds.

\section{Conclusion}

Squalene is a super lubricant with skin compatibility and thermostability. Traditional source of squalene from shark-hunting or oil plant extraction is cost-prohibitive and not sustainable. Microbial fermentation is considered as a promising route to upgrade sugar renewable feedstock to squalene. By reconstituting mevalonate pathway in yeast, a few of studies have achieved considerable amount of squalene. In this work, we reported the engineering of the oleaginous yeast, $Y$. lipolytica, as a potential host for squalene production. We surveyed a number of HMG-CoA reductase and discovered that endogenous HMG-CoA reductase led to the highest squalene improvement. With the recycling of NADPH from the mannitol cycle, the engineered strain (with $\mathrm{MnDH} 2$ overexpression) produced about $180.3 \mathrm{mg} / \mathrm{l}$ and $188.2 \mathrm{mg} / \mathrm{L}$ squalene from glucose or acetate minimal media, respectively. We identified the 
optimal NADPH and acetyl-CoA supply mode. Upon overexpression of squalene synthase, HMG-CoA reductase, mannitol dehydrogenase or ATP-citrate lyase, we further optimized the cultivation conditions. The engineered strain fermented with $\mathrm{C} / \mathrm{N}$ 40:1 media conditioned with PBS buffer with supplementation of $1 \mathrm{mg} / \mathrm{L}$ cerulenin produced about $502.7 \mathrm{mg} / \mathrm{L}$ squalene in shake flaks. The metabolic byproduct citric acid and mannitol level were also profiled, both byproducts were reincorporated into cell metabolism at the late stage of fermentation. This work may serve as a starting point to harness $Y$. lipolytica as an oleaginous yeast factory for costefficient production of squalene or terpene-based chemicals.

\section{Author contributions}

PX conceived the topic and designed the study. HL performed genetic engineering and fermentation experiments. HL and PX wrote the manuscript. PX revised the manuscript.

\section{Conflicts of interests}

A provisional patent has been filed based on the results of this study.

\section{Acknowledgments}

We would like to acknowledge Bill \& Melinda Gates Foundation (grant number OPP1188443) and National Science Foundation (CBET-1805139) for financially supporting this project. The authors would also like to acknowledge the Department of Chemical, Biochemical and Environmental Engineering at University of Maryland Baltimore County for funding support. HL would like to thank the China Scholarship Council for funding support. 


\section{References}

Bae, S.-J., Park, B. G., Kim, B.-G., \& Hahn, J.-S. (2020). Multiplex Gene Disruption by Targeted Base Editing of Yarrowia lipolytica Genome Using Cytidine Deaminase Combined with the CRISPR/Cas9 System. Biotechnology Journal, 15(1), 1900238. doi:10.1002/biot.201900238

Cao, X., Wei, L.-J., Lin, J.-Y., \& Hua, Q. (2017). Enhancing linalool production by engineering oleaginous yeast Yarrowia lipolytica. Bioresour Technol, 245, 1641-1644. doi:https://doi.org/10.1016/j.biortech.2017.06.105

Celińska, E., Ledesma-Amaro, R., Larroude, M., Rossignol, T., Pauthenier, C., \& Nicaud, J.-M. (2017). Golden Gate Assembly system dedicated to complex pathway manipulation in Yarrowia lipolytica. Microbial Biotechnology, 10(2), 450-455. doi:10.1111/1751-7915.12605

Chen, D. C., Beckerich, J. M., \& Gaillardin, C. (1997). One-step transformation of the dimorphic yeast Yarrowia lipolytica. Applied Microbiology and Biotechnology, 48(2), 232-235. doi:10.1007/s002530051043

Dobrowolski, A., Mituła, P., Rymowicz, W., \& Mirończuk, A. M. (2016). Efficient conversion of crude glycerol from various industrial wastes into single cell oil by yeast Yarrowia lipolytica. Bioresour Technol, 207, 237-243.

Egermeier, M., Sauer, M., \& Marx, H. (2019). Golden Gate-based metabolic engineering strategy for wild-type strains of Yarrowia lipolytica. FEMS Microbiology Letters, 366(4). doi:10.1093/femsle/fnz022

El-Ashgar, N. M., El-Basioni, A. I., El-Nahhal, I. M., Zourob, S. M., El-Agez, T. M., \& Taya, S. A. (2012). Solgel thin films immobilized with bromocresol purple $\mathrm{pH}$-sensitive indicator in presence of surfactants. ISRN Analytical Chemistry, 2012.

Gao, S., Tong, Y., Zhu, L., Ge, M., Zhang, Y., Chen, D., . . Yang, S. (2017). Iterative integration of multiplecopy pathway genes in Yarrowia lipolytica for heterologous $\beta$-carotene production. Metabolic Engineering, 41, 192-201. doi:https://doi.org/10.1016/j.ymben.2017.04.004

Gibson, D. G., Young, L., Chuang, R.-Y., Venter, J. C., Hutchison III, C. A., \& Smith, H. O. (2009). Enzymatic assembly of DNA molecules up to several hundred kilobases. Nature methods, 6(5), 343.

Groenewald, M., Boekhout, T., Neuvéglise, C., Gaillardin, C., van Dijck, P. W. M., \& Wyss, M. (2014). Yarrowia lipolytica: Safety assessment of an oleaginous yeast with a great industrial potential. Critical Reviews in Microbiology, 40(3), 187-206. doi:10.3109/1040841X.2013.770386

Han, J. Y., Seo, S. H., Song, J. M., Lee, H., \& Choi, E.-S. (2018). High-level recombinant production of squalene using selected Saccharomyces cerevisiae strains. J Ind Microbiol Biotechnol, 45(4), 239251.

Huang, Y.-Y., Jian, X.-X., Lv, Y.-B., Nian, K.-Q., Gao, Q., Chen, J., . . Hua, Q. (2018). Enhanced squalene biosynthesis in Yarrowia lipolytica based on metabolically engineered acetyl-CoA metabolism. Journal of Biotechnology, 281, 106-114. doi:https://doi.org/10.1016/j.jbiotec.2018.07.001

Jin, C.-C., Zhang, J.-L., Song, H., \& Cao, Y.-X. (2019). Boosting the biosynthesis of betulinic acid and related triterpenoids in Yarrowia lipolytica via multimodular metabolic engineering. Microbial Cell Factories, 18(1), 77. doi:10.1186/s12934-019-1127-8

Larroude, M., Celinska, E., Back, A., Thomas, S., Nicaud, J.-M., \& Ledesma-Amaro, R. (2018). A synthetic biology approach to transform Yarrowia lipolytica into a competitive biotechnological producer of $\beta$-carotene. Biotechnology and Bioengineering, 115(2), 464-472. doi:10.1002/bit.26473

Larroude, M., Park, Y. K., Soudier, P., Kubiak, M., Nicaud, J. M., \& Rossignol, T. (2019). A modular Golden Gate toolkit for Yarrowia lipolytica synthetic biology. Microb Biotechnol, 12(6), 1249-1259. doi:10.1111/1751-7915.13427 
Larroude, M., Trabelsi, H., Nicaud, J.-M., \& Rossignol, T. (2020). A set of Yarrowia lipolytica CRISPR/Cas9 vectors for exploiting wild-type strain diversity. Biotechnology Letters. doi:10.1007/s10529-02002805-4

Liu, G.-S., Li, T., Zhou, W., Jiang, M., Tao, X.-Y., Liu, M., . . Wei, D.-Z. (2020). The yeast peroxisome: A dynamic storage depot and subcellular factory for squalene overproduction. Metabolic Engineering, 57, 151-161. doi:https://doi.org/10.1016/j.ymben.2019.11.001

Liu, H., Fan, J., Wang, C., Li, C., \& Zhou, X. (2019). Enhanced $\beta$-Amyrin Synthesis in Saccharomyces cerevisiae by Coupling An Optimal Acetyl-CoA Supply Pathway. Journal Of Agricultural And Food Chemistry, 67(13), 3723-3732. doi:10.1021/acs.jafc.9b00653

Liu, H., Marsafari, M., Deng, L., \& Xu, P. (2019). Understanding lipogenesis by dynamically profiling transcriptional activity of lipogenic promoters in Yarrowia lipolytica. Applied Microbiology and Biotechnology, 103(7), 3167-3179. doi:10.1007/s00253-019-09664-8

Liu, H., Marsafari, M., Wang, F., Deng, L., \& Xu, P. (2019). Engineering acetyl-CoA metabolic shortcut for eco-friendly production of polyketides triacetic acid lactone in Yarrowia lipolytica. Metabolic Engineering, 56, 60-68. doi:https://doi.org/10.1016/i.ymben.2019.08.017

Lv, Y., Edwards, H., Zhou, J., \& Xu, P. (2019). Combining 26s rDNA and the Cre-loxP System for Iterative Gene Integration and Efficient Marker Curation in Yarrowia lipolytica. ACS Synthetic Biology, 8(3), 568-576. doi:10.1021/acssynbio.8b00535

Lv, Y., Marsafari, M., Koffas, M., Zhou, J., \& Xu, P. (2019). Optimizing Oleaginous Yeast Cell Factories for Flavonoids and Hydroxylated Flavonoids Biosynthesis. ACS Synthetic Biology, 8(11), 2514-2523. doi:10.1021/acssynbio.9b00193

Ma, Y.-R., Wang, K.-F., Wang, W.-J., Ding, Y., Shi, T.-Q., Huang, H., \& Ji, X.-J. (2019). Advances in the metabolic engineering of Yarrowia lipolytica for the production of terpenoids. Bioresour Technol, 281, 449-456. doi:https://doi.org/10.1016/j.biortech.2019.02.116

Marsafari, M., \& Xu, P. (2020). Debottlenecking mevalonate pathway for antimalarial drug precursor amorphadiene biosynthesis in Yarrowia lipolytica. Metabolic Engineering Communications, 10, e00121. doi:https://doi.org/10.1016/j.mec.2019.e00121

Meadows, A. L., Hawkins, K. M., Tsegaye, Y., Antipov, E., Kim, Y., Raetz, L., . . Xu, L. (2016). Rewriting yeast central carbon metabolism for industrial isoprenoid production. Nature, 537(7622), 694.

Minard, K. I., Jennings, G. T., Loftus, T. M., Xuan, D., \& McAlister-Henn, L. (1998). Sources of NADPH and expression of mammalian NADP+-specific isocitrate dehydrogenases in Saccharomyces cerevisiae. J Biol Chem, 273(47), 31486-31493.

Minard, K. I., \& McAlister-Henn, L. (2005). Sources of NADPH in yeast vary with carbon source. J Biol Chem, 280, 39890-39896.

Moser, S., \& Pichler, H. (2019). Identifying and engineering the ideal microbial terpenoid production host. Applied Microbiology and Biotechnology, 103(14), 5501-5516. doi:10.1007/s00253-01909892-y

Nowrousian, M., Kück, U., Loser, K., \& Weltring, K.-M. (2000). The fungal acl1 and acl2 genes encode two polypeptides with homology to the $\mathrm{N}$-and $\mathrm{C}$-terminal parts of the animal ATP citrate lyase polypeptide. Current Genetics, 37(3), 189-193.

Palmer, C. M., Miller, K. K., Nguyen, A., \& Alper, H. S. (2020). Engineering 4-coumaroyl-CoA derived polyketide production in Yarrowia lipolytica through a $\beta$-oxidation mediated strategy. Metabolic Engineering, 57, 174-181. doi:https://doi.org/10.1016/j.ymben.2019.11.006

Palsuledesai, C. C., \& Distefano, M. D. (2015). Protein Prenylation: Enzymes, Therapeutics, and Biotechnology Applications. ACS Chemical Biology, 10(1), 51-62. doi:10.1021/cb500791f

Polakowski, T., Stahl, U., \& Lang, C. (1998). Overexpression of a cytosolic hydroxymethylglutaryl-CoA reductase leads to squalene accumulation in yeast. Appl Microbiol Biotechnol, 49(1), 66-71. 
Qiao, K., Wasylenko, T. M., Zhou, K., Xu, P., \& Stephanopoulos, G. (2017). Lipid production in Yarrowia lipolytica is maximized by engineering cytosolic redox metabolism. Nat Biotechnol, 35(2), 173177. doi:10.1038/nbt.3763

Rakicka, M., Biegalska, A., Rymowicz, W., Dobrowolski, A., \& Mirończuk, A. M. (2017). Polyol production from waste materials by genetically modified Yarrowia lipolytica. Bioresour Technol, 243, 393399.

RODWELL, V. W., NORDSTROM, J. L., \& MITSCHELEN, J. J. (1976). Regulation of HMG-CoA reductase. In Advances in lipid research (Vol. 14, pp. 1-74): Elsevier.

Seip, J., Jackson, R., He, H., Zhu, Q., \& Hong, S.-P. (2013). Snf1 is a regulator of lipid accumulation in Yarrowia lipolytica. Appl. Environ. Microbiol., 79(23), 7360-7370.

Shimano, H. (2001). Sterol regulatory element-binding proteins (SREBPs): transcriptional regulators of lipid synthetic genes. Progress in Lipid Research, 40(6), 439-452. doi:https://doi.org/10.1016/S0163-7827(01)00010-8

Spanova, M., \& Daum, G. (2011). Squalene - biochemistry, molecular biology, process biotechnology, and applications. European Journal of Lipid Science and Technology, 113(11), 1299-1320. doi:10.1002/ejlt.201100203

Szabo, R. (1999). Dimorphism inYarrowia lipolytica: filament formation is suppressed by nitrogen starvation and inhibition of respiration. Folia Microbiologica, 44(1), 19-24.

Thompson, A., Kwak, S., \& Jin, Y.-S. (2014). Squalene production using Saccharomyces cerevisiae. i-ACES, 1(1), 57-63.

van Rossum, H. M., Kozak, B. U., Pronk, J. T., \& van Maris, A. J. (2016). Engineering cytosolic acetylcoenzyme A supply in Saccharomyces cerevisiae: pathway stoichiometry, free-energy conservation and redox-cofactor balancing. Metabolic Engineering, 36, 99-115.

Wagner, J. M., Williams, E. V., \& Alper, H. S. (2018). Developing a piggyBac Transposon System and Compatible Selection Markers for Insertional Mutagenesis and Genome Engineering in Yarrowia lipolytica. Biotechnology Journal, 13(5), 1800022. doi:10.1002/biot.201800022

Wasylenko, T. M., Ahn, W. S., \& Stephanopoulos, G. (2015). The oxidative pentose phosphate pathway is the primary source of NADPH for lipid overproduction from glucose in Yarrowia lipolytica. Metab Eng, 30.

Wong, L., Engel, J., Jin, E., Holdridge, B., \& Xu, P. (2017). YaliBricks, a versatile genetic toolkit for streamlined and rapid pathway engineering in Yarrowia lipolytica. Metabolic Engineering Communications, 5(Supplement C), 68-77. doi:https://doi.org/10.1016/j.meteno.2017.09.001

Wong, L., Holdridge, B., Engel, J., \& Xu, P. (2019). Genetic Tools for Streamlined and Accelerated Pathway Engineering in Yarrowia lipolytica. In C. N. S. Santos \& P. K. Ajikumar (Eds.), Microbial Metabolic Engineering: Methods and Protocols (pp. 155-177). New York, NY: Springer New York.

Xie, X., \& Tang, Y. (2007). Efficient synthesis of simvastatin by use of whole-cell biocatalysis. Applied and Environmental Microbiology, 2054-2060. doi:DOI 10.1128/AEM.02820-06

Xu, J., Liu, N., Qiao, K., Vogg, S., \& Stephanopoulos, G. (2017). Application of metabolic controls for the maximization of lipid production in semicontinuous fermentation. Proceedings of the National Academy of Sciences, 114(27), E5308-E5316.

Xu, P., Qiao, K., Ahn, W. S., \& Stephanopoulos, G. (2016). Engineering Yarrowia lipolytica as a platform for synthesis of drop-in transportation fuels and oleochemicals. Proceedings of the National Academy of Sciences, 113(39), 10848-10853.

Xu, P., Qiao, K., \& Stephanopoulos, G. (2017). Engineering oxidative stress defense pathways to build a robust lipid production platform in Yarrowia lipolytica. Biotechnol Bioeng, 114(7), 1521-1530.

Yang, Z., Edwards, H., \& Xu, P. (2020). CRISPR-Cas12a/Cpf1-assisted precise, efficient and multiplexed genome-editing in Yarrowia lipolytica. Metabolic Engineering Communications, 10, e00112. doi:https://doi.org/10.1016/j.mec.2019.e00112 
bioRxiv preprint doi: https://doi.org/10.1101/2020.05.03.075259; this version posted May 4, 2020. The copyright holder for this preprint (which

was not certified by peer review) is the author/funder, who has granted bioRxiv a license to display the preprint in perpetuity. It is made available under aCC-BY-NC-ND 4.0 International license.

Zhang, M., Galdieri, L., \& Vancura, A. (2013). The yeast AMPK homolog SNF1 regulates acetyl coenzyme A homeostasis and histone acetylation. Molecular and Cellular Biology, 33(23), 4701-4717. 


\section{Figures captions}

Fig. 1 Metabolic pathway for squalene synthesis in oleaginous yeast. MnDH, mannitol dehydrogenase; HXK, hexokinase; MAE1, malic enzyme; ACL2, ATP citrate lyase; IDP2, cytosolic NADP-specific isocitrate dehydrogenase; UGA2, succinate semialdehyde dehydrogenase; PYC1, pyruvate carboxylase; PDC1, pyruvate decarboxylase; ALD, aldehyde dehydrogenase; PDH, pyruvate dehydrogenase complex; ACS, acetyl-CoA synthase; FAS1 and FAS2, fatty acid synthase; ACC1, acetyl-CoA carboxylase; HMG, HMG-CoA reductase; Erg 10, acetoacetyl-CoA thiolase; Erg 12, mevalonate kinase; Erg 8, phosphomevalonate kinase; MVD, mevalonate pyrophosphate decarboxylase; Erg 20, farnesyl pyrophosphate synthetase; GPS, geranyl pyrophosphate synthase; SQS, squalene synthase.

Fig. 2 Comparison of different HMG-CoA reductase and identification of rate-limiting steps of endogenous mevalonate pathway in Y. lipolytica.

Fig. 3 Enhancement of NAPDH and acetyl-CoA precursor pathways to improve squalene production. ScPDC1, pyruvate decarboxylase from S. cerevisiae; EcPuuc, aldehyde dehydrogenase from E. coli. Other genes are native genes from Y. lipolytica and detailed gene annotation could be found in Fig. 1.

Fig. 4 Improving squalene production by controlling $\mathrm{pH}$ and $\mathrm{C} / \mathrm{N}$ ratio with cerulenin supplementation in glucose-minimal media. Fermentation profile of glucose consumption, mannitol, dry cell weight, citric acid and squalene accumulation for strain HLYaliSO2 cultivated in glucose-minimal media conditioned with PBS buffer and supplemented with $1 \mathrm{mg} / \mathrm{L}$ cerulenin 
bioRxiv preprint doi: https://doi.org/10.1101/2020.05.03.075259; this version posted May 4, 2020. The copyright holder for this preprint (which

was not certified by peer review) is the author/funder, who has granted bioRxiv a license to display the preprint in perpetuity. It is made available under aCC-BY-NC-ND 4.0 International license.

(A). Fermentation profile of glucose consumption, mannitol, dry cell weight, citric acid and squalene accumulation for strain HLYaliSO2 cultivated in glucose-minimal media conditioned with PBS buffer, supplemented with $1 \mathrm{mg} / \mathrm{L}$ cerulenin and C/N ratio 40:1 (B). 


\section{Figures}

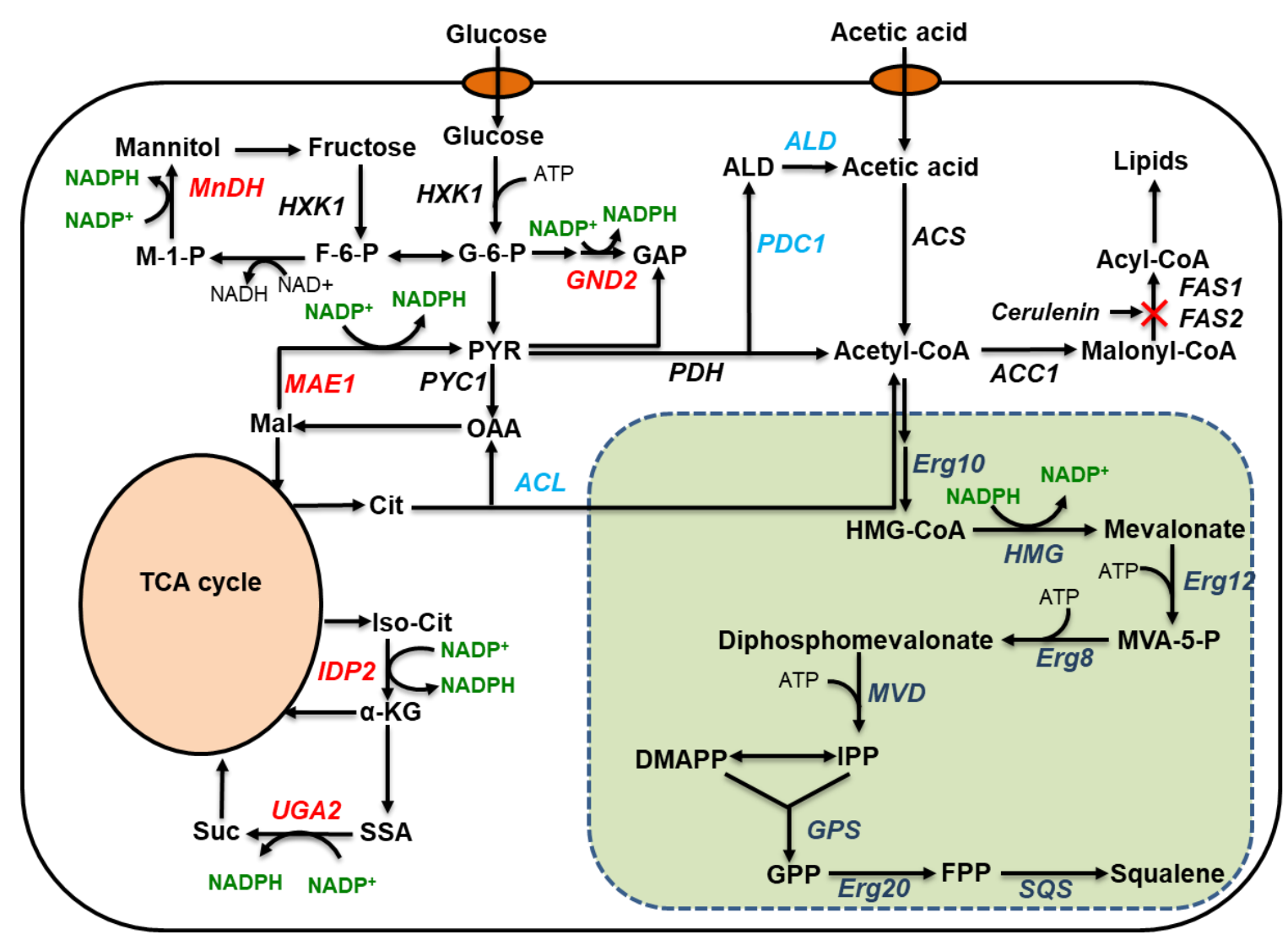

Fig. 1 

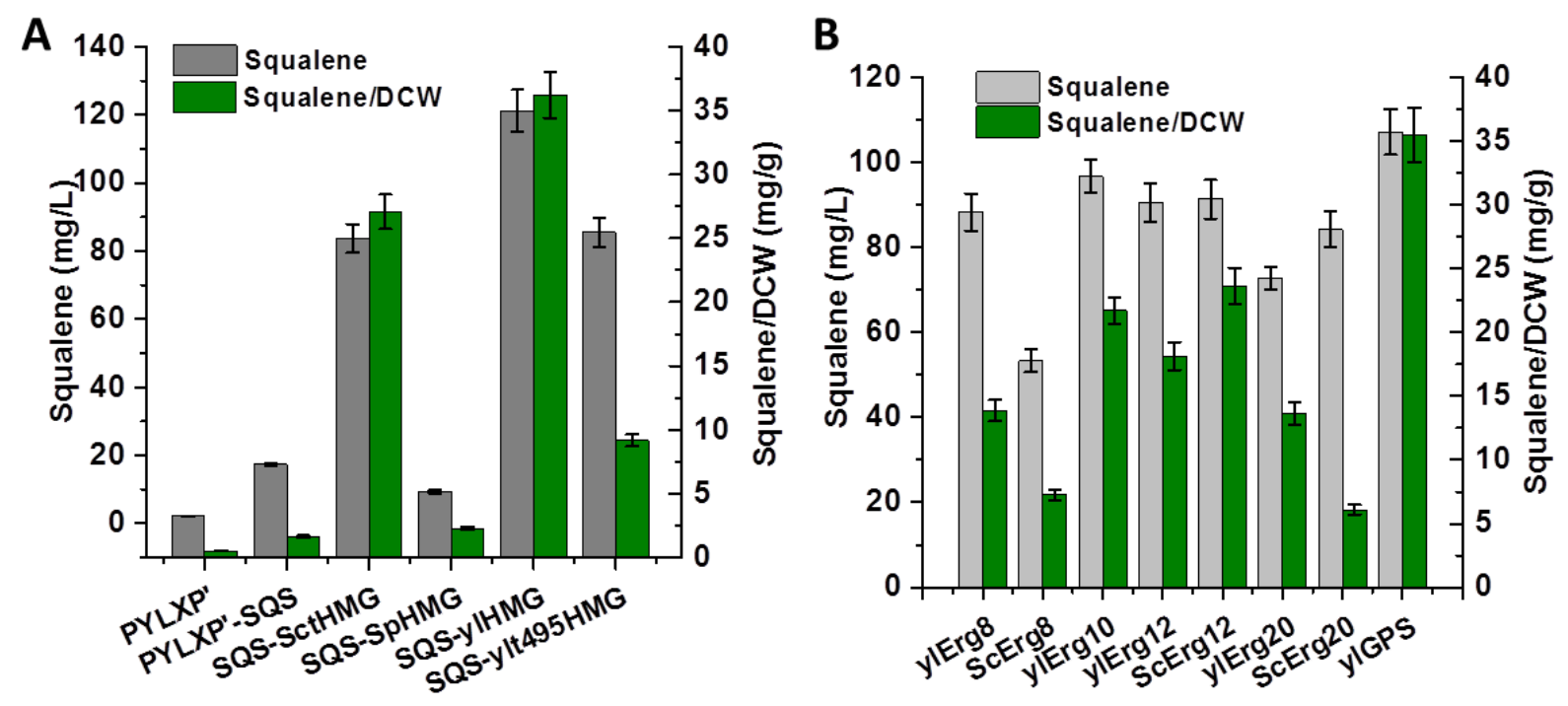

Fig. 2
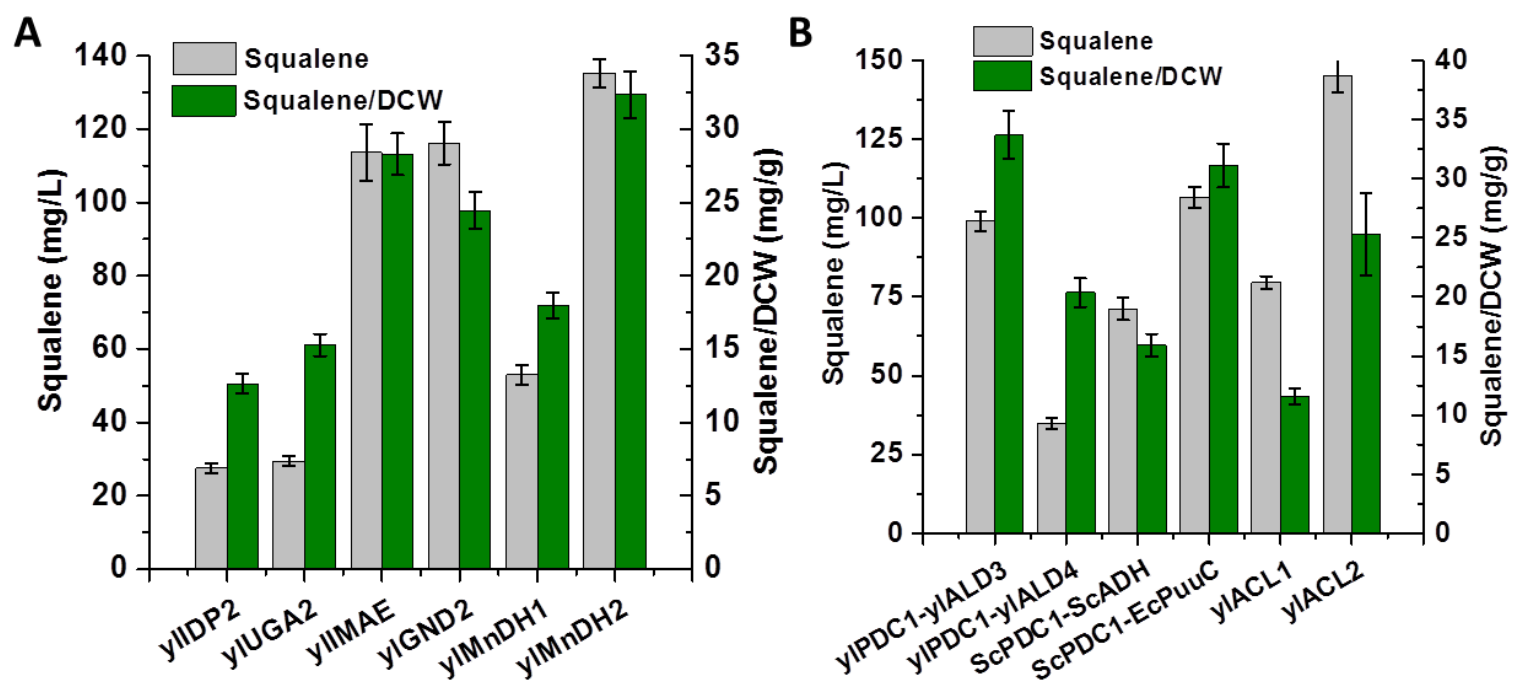

Fig. 3 
A

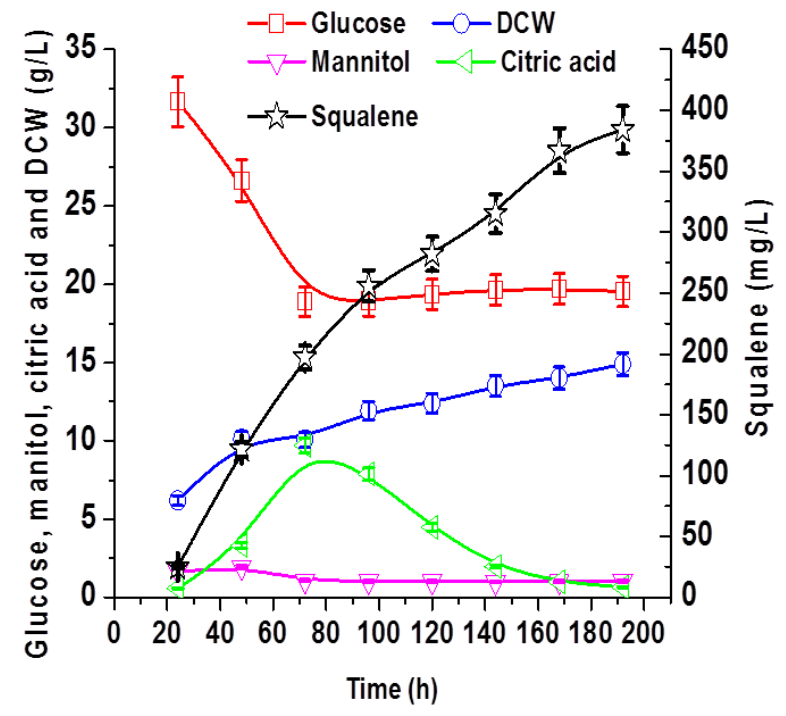

B

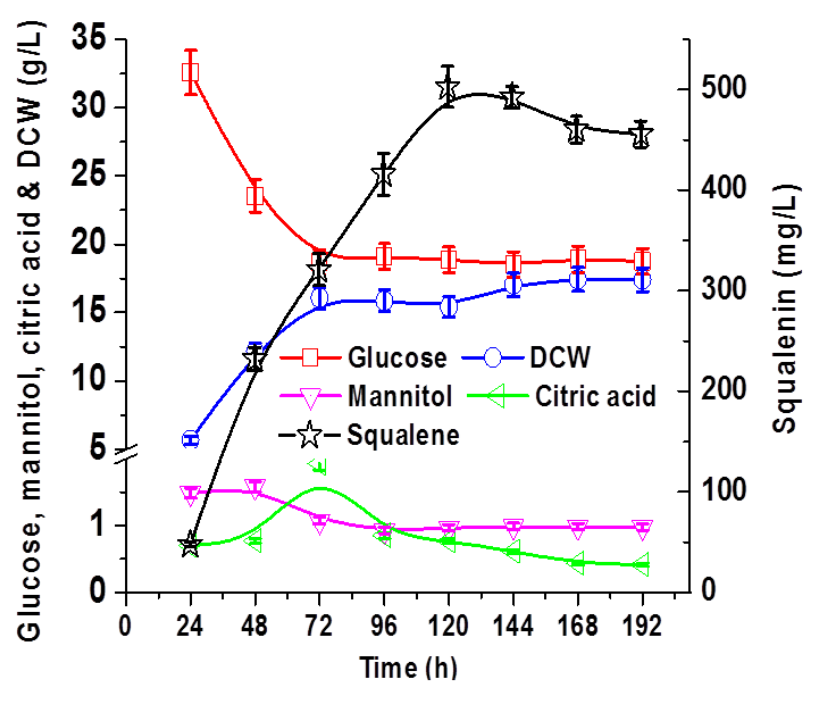

Fig. 4 